\section{Posterior cingulate cortex in Alzheimer's disease}

SIR-In the living brain of patients with Alzheimer's disease, positron emission tomography with ${ }^{18} \mathrm{~F}$-fluorodeoxyglucose (FDG) reveals characteristic hypometabolism in neocortical structures, especially in parietal, temporal, and frontal association cortex, the same locations where co-existing neuronal degeneration is revealed postmortem. ${ }^{1,2}$ Neuronal degeneration is also found in the limbic system, a structure closely related to memory and other cognitive processes, but corresponding metabolic abnormalities have not been emphasised by in-vivo imaging.

Using new image-processing technology, we found severe metabolic reduction in the posterior cingulate cortex in Alzheimer's disease. FDG images from positron emission tomography in 37 patients with probable Alzheimer's disease and 22 normal controls were transformed anatomically to the uniform shape of a standard stereotactic atlas brain while preserving regional metabolic information. ${ }^{3}$ Metabolic activities of lateral and medial brain structures were extracted from the images, averaged within each group, and compared with $t$ statistics. Statistical surface-projection maps demonstrated metabolic reduction in the posterior cingulate cortex, which was as significant as that in the parietotemporal neocortex (figure). The metabolic reduction in the posterior cingulate cortex correlated most closely with reductions in the medial parietal cortex, followed by those in the lateral parietal cortex. In addition, the metabolic reduction in the posterior cingulate cortex was present in 3 patients with Alzheimer's disease who were only questionably demented at the time of scanning.

The metabolic reduction in the posterior cingulate cortex is strong evidence of abnormalities of energy metabolism involving the limbic system and is consistent with underlying neuronal degeneration in the posterior cingulate cortex in Alzheimer's disease. ${ }^{2}$ Metabolic correlation between the posterior cingulate cortex and the parietal cortex may be due to neuronal interconnections. ${ }^{*}$ Interestingly, the anterior cingulate cortex which has distinct connections and

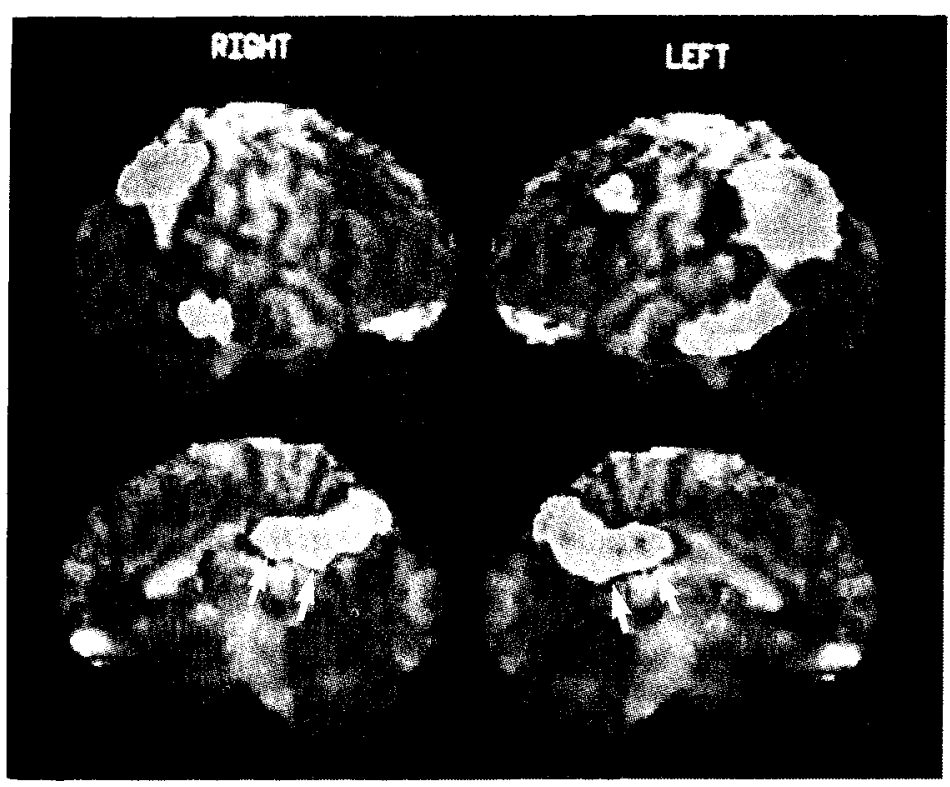

Figure: Three-dimensional views of cerebral metabolic reduction in Alzheimer's disease

Areas of statistically significant metabolic reduction $(p<0.05$ adjusted for multiple comparisons) superimposed on brain surface images of each hemisphere. Colour scale indicates degree of significance

(red>yellow > green). On lateral views (top), parietal, temporal, and frontal neocortex show hypometabolısm. Note concordance between pattern of hypometabolic regions and that of neuronal degeneration previously reported. ${ }^{1}$ On medial views (bottom), posterior cingulate cortex (arrows), cinguloparietal transition area, and medial parietal cortex show metabolıc reduction. No such abnormalities were present in anterior cingulate cortex. behavioural attributes from those of the posterior cingulate cortex, was spared, as it is histopathologically. ${ }^{2}$ The cause of abnormal energy metabolism in the posterior cingulate circuit and its functional significance in cognitive dysfunctions in Alzheimer's disease need further investigation. Detection of metabolic and possibly neurochemical abnormalities ${ }^{5}$ in the posterior cingulate cortex could enhance accuracy in early in-vivo diagnosis of Alzheimer's disease.

This study was supported in part by grants RO1-NS24896 from the National Institutes of Health and DE-FG02-87-ER60561 from the US Department of Energy.

Satoshi Minoshima, Norman L Foster, David E Kuhl Departments of Internal Medicine and of Radiology, Division of Nuclear Medicine, University of Michigan, Ann Arbor, MI 48109, USA

1 Friedland RP, Brun A, Budinger TF. Pathological and positron emission tomographic correlations in Alzheimer's disease. Lancet 1985; i: 228 .

2 Brun A, Gustafson L. Distribution of cerebral degeneration in Alzheimer's disease. Arch Psychiat Nervenkr 1976; 223: 15-33.

3 Minoshima S, Koeppe RA, Frey KA, Kuhl DE. Anatomical standardization: linear scaling and non-linear warping of functional brain images. $\mathcal{F ~} \mathrm{Nucl}$ Med $1994 ; 35$ : 1528-37.

4 Baleydire $\mathrm{C}$, Mauguiere $\mathrm{F}$. The duality of the cingulate gyrus in monkey: neuroanatomical study and functional hypothesis. Brain 1980; 103: 525-54.

5 Kuhl DE, Koeppe RA, Fessler JA, et al. In vivo mapping of cholinergic neurons in the human brain using SPECT and IBVM. F Nucl Med 1994; 35: 405-10.

\section{The global challenge of tuberculosis}

SIR-In your July 30 editorial on tuberculosis you state that by far the largest share of tuberculosis cases is in developing countries and that "estimates suggest that fewer than half the patients ... are in contact with treatment services". Further on, you review the topics tackled by the new "flurry of research after two decades of neglect": development of new drugs, rapid molecular diagnostic techniques. As others have done, ${ }^{1}$ you give the impression that further technological advances will provide the answer to this global challenge. We consider this is a rather narrow basis for selecting research priorities.

Case-finding and treatment remain the cornerstone of tuberculosis control. Improved diagnostic and therapeutic techniques may indeed help to reduce some of the leakage between occurrence of tuberculosis and cure. However, as $M$ Piot of WHO pointed out in the early 1960 s, there are a series of potential bottlenecks. Patients with tuberculosis may or may not contact treatment services; those who do so might not be identified as cases of tuberculosis; those that are identified may not be prescribed treatment; those that are prescribed treatment might not be able to obtain it; those that obtain treatment may fail to complete treatment; and treatment may fail to cure some of those who complete the prescribed regimen. Successful cure can be achieved only if these possible points of leakage are overcome.

Specifically, increasing treatment efficacy will only have a marginal effect if major leakage occurs at all the preceding steps; this seems to be the case not only in developing countries, but also in parts of the industrialised world. Surprisingly little headway has been made over past decades in issues such as integration of tuberculosis control activities, the development of strategies to improve patient compliance and ensure continuity of care, or the crucial issue of financial access to treatment.

I hope that The Lancet's September, 1995, conference in Washington will not be devoted exclusively to the biological aspects of the resurgence of tuberculosis. Behavioural and 